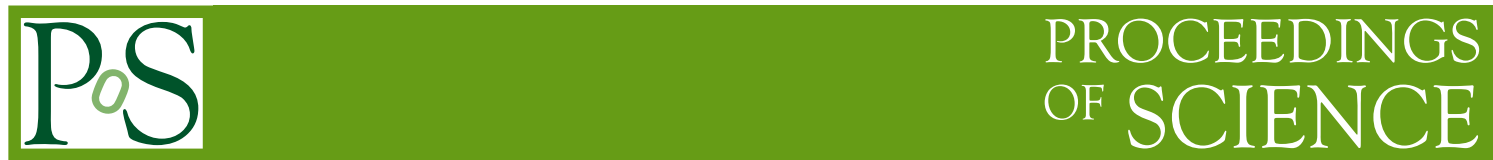

\title{
Color superconductivity in ultra-dense quark matter
}

\section{Mark Alford*}

Physics Department, Washington University, St. Louis, MO 63130, USA

E-mail: alford@wuphys.wustl.edu,

\begin{abstract}
At ultra-high density, matter is expected to form a degenerate Fermi gas of quarks in which there is a condensate of Cooper pairs of quarks near the Fermi surface. This phenomenon is called color superconductivity. In these proceedings I review the underlying physics of color superconductivity and our current understanding of the possible phases of real-world quark matter. Then I consider how lattice gauge theorists would proceed to investigate the phase structure of dense quark matter if it were possible to perform the path integral numerically, i.e. if the sign problem had been solved.
\end{abstract}

XXIVth International Symposium on Lattice Field Theory

July 23-28, 2006

Tucson, Arizona, USA

\footnotetext{
${ }^{*}$ Speaker.
} 


\section{Introduction}

The exploration of the phase diagram of matter at ultra-high temperature or density is an area of great interest and activity, both on the experimental and theoretical fronts. Heavy-ion colliders such as the SPS at CERN and RHIC at Brookhaven have probed the high-temperature region, creating and studying the properties of quark matter with very high energy density and very low baryon number density similar to the fluid which filled the universe for the first microseconds after the big bang. Lattice gauge theory calculations have located the critical temperature and shown that the quark gluon plasma (QGP) is still strongly coupled at temperatures in heavy ion collisions. There has also been striking progress in performing lattice calculations in the "not too dense" region, $\mu<T$, In these proceedings, however, I discuss a different part of the phase diagram, the low-temperature high-density region where $T \ll \mu$. Here there are few experimental constraints, and the sign problem has blocked lattice QCD calculations. However, as I will explain, we have reasons to expect interesting phase structure.

\subsection{Review of color superconductivity}

QCD is asymptotically free- the interaction becomes weaker as the momentum grows - so at sufficiently high density and low temperature, there is a Fermi surface of almost free quarks. The interactions between quarks near the Fermi surface are certainly attractive in some channels (quarks bind together to form baryons) and it was shown by Bardeen, Cooper, and Schrieffer (BCS) [1] that if there is any channel in which the interaction is attractive, then there is a state of lower free energy than a simple Fermi surface. That state arises from a complicated coherent superposition of pairs of particles (and holes) - “Cooper pairs".

We can understand the BCS mechanism in an intuitive way as follows. The Helmholtz free energy is $F=E-\mu N$, where $E$ is the total energy of the system, $\mu$ is the chemical potential, and $N$ is the number of fermions. The Fermi surface is defined by a Fermi energy $E_{F}=\mu$, at which the free energy is minimized, so adding or subtracting a single particle costs zero free energy. Now switch on a weak attractive interaction. It costs no free energy to add a pair of particles (or holes), and the attractive interaction between them then lowers the free energy of the system. Many such pairs will therefore be created in the modes near the Fermi surface, and these pairs, being bosonic, will form a condensate. The ground state will be a superposition of states with all numbers of pairs, breaking the fermion number symmetry.

Since pairs of quarks cannot be color singlets, the resulting condensate will break the local color symmetry $S U(3)_{\text {color }}$. We call this "color superconductivity" [2]. Note that the quark pairs play the same role here as the Higgs particle does in the standard model: the color-superconducting phase can be thought of as the Higgs phase of QCD.

\section{The phases of quark matter}

Quarks, unlike electrons, have color and flavor as well as spin degrees of freedom, so many different patterns of pairing are possible. This leads us to expect a rich structure of different color superconducting phases in quark matter at very high density. 
Conjectured form

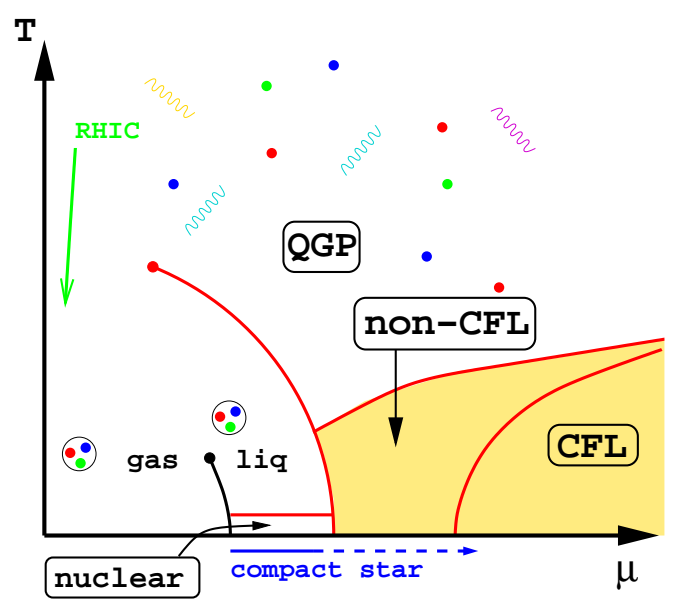

$\underline{\text { NJL calculation }}$

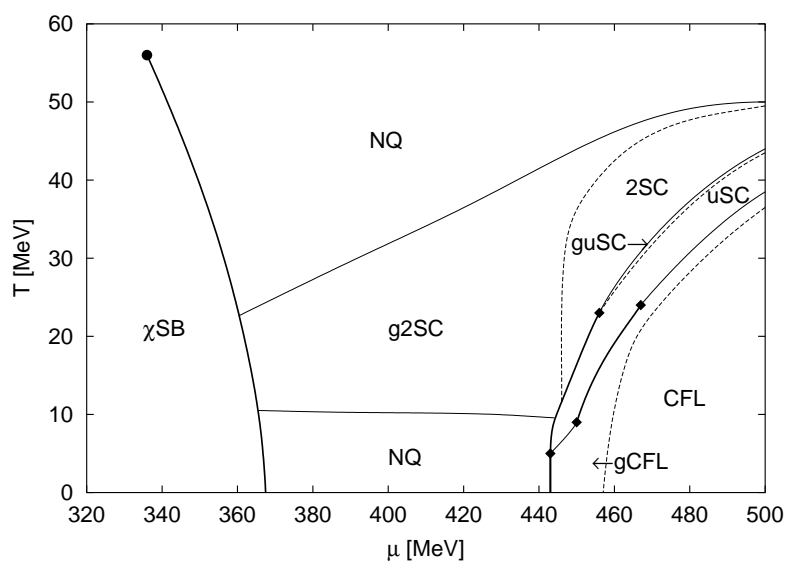

Figure 1: On the left, the conjectured form of the phase diagram for matter at ultra-high density and temperature. On the right, the result of a calculation using an NJL model [3]. At high density the model has a rich structure of color-superconducting phases. Note that the gapless phases ("gCFL", "g2SC" etc) are unstable (see text).

In the real world there are two light quark flavors, the up $(u)$ and down $(d)$, with masses $\lesssim 5 \mathrm{MeV}$, and a medium-weight flavor, the strange (s) quark, with mass $\sim 90 \mathrm{MeV}$. (Their effective "constituent" masses in dense matter may be much larger.) The strange quark therefore plays a crucial role in the phases of QCD. Fig. 1 shows a conjectured phase diagram for QCD, and also a calculated phase diagram obtained using a Nambu-Jona-Lasinio model of QCD [3]. In both cases, along the horizontal axis the temperature is zero, and the density rises from the onset of nuclear matter through the transition to quark matter. Compact stars are in this region of the phase diagram, although it is not known whether their cores are dense enough to reach the quark matter phase. Along the vertical axis the temperature rises, taking us through the crossover from a hadronic gas to the quark gluon plasma. This is the regime explored by high-energy heavy-ion colliders.

At the highest densities we find the CFL phase, in which the strange quark participates symmetrically with the up and down quarks in Cooper pairing - this is described in more detail below. It is not yet clear what happens at intermediate density, and in the next section I will briefly survey the phases that have been hypothesized to occur there. The Nambu-Jona-Lasinio model is only a semi-quantitative guide to the possible behavior of QCD, so its predictions shown in Fig. 1 should be taken as purely illustrative.

\subsection{Highest density: Color-flavor locking (CFL)}

It is by now well-established that at sufficiently high densities, where the up, down and strange quarks can be treated on an equal footing and the disruptive effects of the strange quark mass can be neglected, quark matter is in the color-flavor locked (CFL) phase, in which quarks of all three colors and all three flavors form conventional Cooper pairs with zero total momentum, and all fermionic excitations are gapped, with the gap parameter $\Delta_{0} \sim 10-100 \mathrm{MeV}[4,2]$. This has been confirmed by both NJL $[4,5]$ and gluon-mediated interaction calculations [6]. The CFL pairing 
pattern is

$$
\begin{aligned}
& \left\langle q_{i}^{\alpha} C \gamma_{5} q_{j}^{\beta}\right\rangle_{1 P I} \propto(\kappa+1) \delta_{i}^{\alpha} \delta_{j}^{\beta}+(\kappa-1) \delta_{j}^{\alpha} \delta_{i}^{\beta}=\varepsilon^{\alpha \beta N} \varepsilon_{i j N}+\kappa(\cdots) \\
& {\left[S U(3)_{\text {color }}\right] \times \underbrace{S U(3)_{L} \times S U(3)_{R}}_{\supset\left[U(1)_{Q}\right]} \times U(1)_{B} \rightarrow \underbrace{S U(3)_{C+L+R}}_{\supset\left[U(1)_{\tilde{Q}}\right]} \times \mathbb{Z}_{2}}
\end{aligned}
$$

Color indices $\alpha, \beta$ and flavor indices $i, j$ run from 1 to 3 , Dirac indices are suppressed, and $C$ is the Dirac charge-conjugation matrix. The term multiplied by $\kappa$ corresponds to pairing in the $\left(\mathbf{6}_{S}, \mathbf{6}_{S}\right)$, which although not energetically favored breaks no additional symmetries and so $\kappa$ is in general small but not zero $[4,6,7,8]$. The Kronecker deltas connect color indices with flavor indices, so that the condensate is not invariant under color rotations, nor under flavor rotations, but only under simultaneous, equal and opposite, color and flavor rotations. Since color is only a vector symmetry, this condensate is only invariant under vector flavor+color rotations, and breaks chiral symmetry. The features of the CFL pattern of condensation are

- The color gauge group is completely broken. All eight gluons become massive. This ensures that there are no infrared divergences associated with gluon propagators.

- All the quark modes are gapped. The nine quasiquarks (three colors times three flavors) fall into an $\mathbf{8} \oplus \mathbf{1}$ of the unbroken global $S U(3)$, so there are two gap parameters. The singlet has a larger gap than the octet.

- A rotated electromagnetism (“ $\tilde{Q}$ ”) survives unbroken. Its gauge boson is a combination of the original photon and one of the gluons.

- Two global symmetries are broken, the chiral symmetry and baryon number, so there are two gauge-invariant order parameters that distinguish the CFL phase from the QGP, and corresponding Goldstone bosons which are long-wavelength disturbances of the order parameter. When the light quark mass is non-zero it explicitly breaks the chiral symmetry and gives a mass to the chiral Goldstone octet, but the CFL phase is still a superfluid, distinguished by its spontaneous breaking of baryon number.

- The symmetries of the 3-flavor CFL phase are the same as those one might expect for 3-flavor hypernuclear matter [5], so it is possible that there is no phase transition between them.

\section{Real-world intermediate-density quark matter}

\subsection{Stresses on the CFL phase}

The CFL phase is characterized by pairing between different flavors and different colors of quarks. This is favored because the QCD interaction between two quarks is most attractive in the channel that is antisymmetric in color (the $\overline{\mathbf{3}}$ ), and pairing tends to be stronger in channels that do not break rotational symmetry $[9,10,11,12,13]$, so we expect the pairing to be a spin singlet, i.e. antisymmetric in spin. Fermionic antisymmetry of the Cooper pair wavefunction then forces the Cooper pair to be antisymmetric in flavor.

Pairing between different colors/flavors can occur easily when they all have the same chemical potentials and Fermi momenta. This is the situation at very high density, where the strange quark mass is negligible. However, in a real compact star we must take into account the forces 
Unpaired

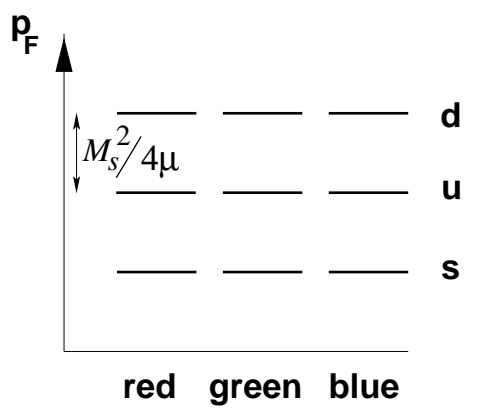

2SC pairing

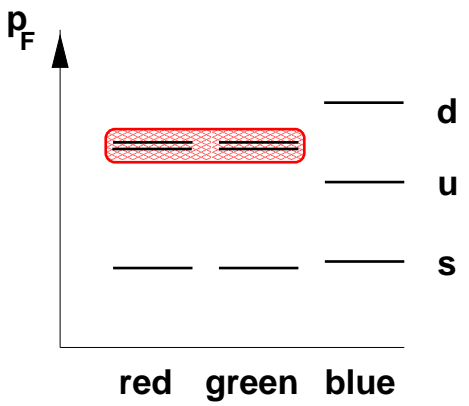

CFL pairing

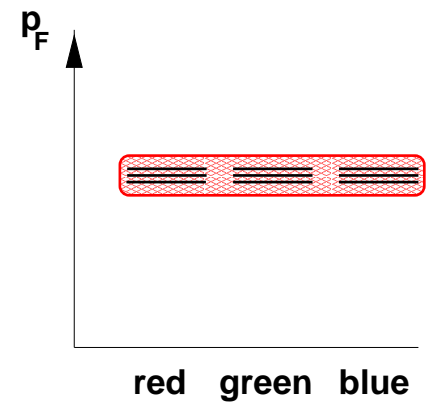

Figure 2: Illustration of the splitting apart of Fermi momenta of the various colors and flavors of quarks. In the unpaired phase, requirements of neutrality and weak interaction equilibration cause separation of the Fermi momenta of the various flavors. In the 2SC phase, up and down quarks of two colors pair, locking their Fermi momenta together. In the CFL phase, all colors and flavors pair and have a common Fermi momentum.

that try to split those Fermi momenta apart, imposing an energy cost on cross-species pairing. We must require electromagnetic and color neutrality $[14,15]$ (possibly via mixing of oppositelycharged phases), allow for equilibration under the weak interaction, and include a realistic mass for the strange quark. These factors cause the different colors and flavors to have different chemical potentials, and this imposes a stress on cross-species pairing such as occurs in the CFL pairing pattern. This is illustrated in Fig. 2, which shows the Fermi momenta of the different species of quarks. In the unpaired phase, the strange quarks have a lower Fermi momentum because they are heavier, and to maintain electrical neutrality the number of down quarks is correspondingly increased (electrons are also present, but play a small role in maintaining neutrality). To lowest order in the strange quark mass, the separation between the Fermi momenta is $M_{s}^{2} /(4 \mu)$, so the splitting is more pronounced at lower density. If the attraction between quarks is sufficiently strong, color superconductivity can overcome this splitting of the Fermi momenta. In the $2 \mathrm{SC}$ phase $[16,17]$, the up and down quarks of two of the colors undergo Cooper pairing, which locks their Fermi momenta together. The pairing will only occur if the energy released by the formation of the condensate is greater than the energy cost of moving the quark Fermi surfaces away from their "natural" positions in the unpaired phase. To lowest order in $M_{s}, 2 \mathrm{SC}$ pairing is favored relative to the unpaired phase when the smearing of the Fermi surface due to Cooper pairing is greater than the splitting, i.e. when $\Delta_{2 \mathrm{SC}}>M_{s}^{2} /(4 \mu)$ [15] (this estimate assumes that contributions to the free energy from the chiral condensate are the same in both phases). In the CFL phase, the pairing is extended to all colors and flavors, which are then locked together with a common Fermi momentum, and the criterion for pairing to occur turns out to be the same, $\Delta_{\mathrm{CFL}}>M_{s}^{2} /(4 \mu)$.

At ultra-high density the splitting between the Fermi momenta becomes negligible, and the CFL phase is favored. However, as the density drops to values that might realistically occur in the core of a neutron star, the value of $M_{s}^{2} /(4 \mu)$ rises to tens of $\mathrm{MeV}$, which is of the same order as the expected pairing gap $\Delta$ in the $2 \mathrm{SC}$ and CFL phases. Thus as the density decreases we expect the CFL pairing pattern to be distorted, and then to be replaced by some other pattern. NJL model calculations [18, 19, 20, 3] find that if the attractive interaction is very strong (so that 
$\Delta_{C F L} \sim 100 \mathrm{MeV}$ where $\Delta_{C F L}$ is what the CFL gap would be at $\mu \sim 500 \mathrm{MeV}$ if $M_{s}$ were zero) then the CFL phase survives all the way down to the transition to nuclear matter. If it is a little less strong then there may be an interval of $2 \mathrm{SC}$ phase but in general the $2 \mathrm{SC}$ phase does not offer a better compromise between pairing and Fermi surface splitting [15, 21]. A comprehensive survey of possible BCS pairing patterns shows that all of them suffer from the stress of Fermi surface splitting [22], so in the intermediate-density region more exotic phases are expected. In the next few subsections we give a quick overview of the expected phases of real-world quark matter at intermediate density. We restrict our discussion to zero temperature because the critical temperatures for most of the phases that we discuss are expected to be of order $10 \mathrm{MeV}$ or higher, and the core temperature of a neutron star is believed to drop below this value within minutes (if not seconds) of its creation in a supernova.

\subsection{Kaon condensation: the CFL- $K^{0}$ phase}

Bedaque and Schäfer [23] showed that when the stress is not too large (high density), it may simply modify the CFL pairing pattern by inducing a flavor rotation of the condensate which can be interpreted as a condensate of " $K^{0}$ " mesons, i.e. the neutral anti-strange Goldstone bosons associated with the chiral symmetry breaking. This is the "CFL- $K^{0}$ " phase, which breaks isospin. The $K^{0}$ condensate can easily be suppressed by instanton effects [24], but if these are ignored then the kaon condensation occurs for $M_{s} \gtrsim m^{1 / 3} \Delta^{2 / 3}$ for light $(u$ and $d$ ) quarks of mass $m$. This was demonstrated using an effective theory of the Goldstone bosons, but with some effort can also be seen in an NJL calculation [25, 26].

\subsection{The unstable gapless phases}

The NJL analysis shown in Fig. 1 predicts that at densities too low for CFL pairing there will be gapless phases ("gCFL",'g2SC", etc). This can be understood by a rough quantitative analysis that involves expanding in powers of $M_{s} / \mu$ and $\Delta / \mu$, and ignoring the fact that the effective strange quark mass may be different in different phases [15]. Such an analysis shows that as we come down in density we find a transition at $\mu \approx \frac{1}{2} M_{s}^{2} / \Delta_{C F L}$ from CFL to another phase, the gapless CFL phase (gCFL) [27]. The underlying physics here is that when $\mu<\frac{1}{2} M_{s}^{2} \Delta_{C F L}$ it becomes energetically favorable to convert a $g s$ quark near the common Fermi momentum into a $b d$ quark, breaking the Cooper pairing over a range of momenta in that channel. The free energies of the competing phases in an NJL model are shown in Fig. 3: The gCFL phase takes over from CFL at $M_{s}^{2} / \mu \approx 2 \Delta_{C F L}$, and remains favored beyond the value $M_{s}^{2} / \mu \approx 4 \Delta_{C F L}$ at which the CFL phase would become unfavored.

However, it turns out that the gapless phases are unstable. The instability of the gCFL phase was established in Refs. [28, 29] after an analogous instability in the gapless 2SC phase had been discovered $[30,31]$. The instability manifests itself in an imaginary Meissner mass $M_{M}$ for some of the gluons. $M_{M}^{2}$ is the low-momentum current-current two-point function, and $M_{M}^{2} /\left(g^{2} \Delta^{2}\right)$ (where the strong interaction coupling is $g$ ) is the coefficient of the gradient term in the effective theory of small fluctuations around the ground-state condensate. The fact that we find a negative value when the quasiparticles are gapless indicates an instability towards spontaneous breaking of translational invariance [32, 33, 34, 35, 36]. Calculations in a simple two-species model [37] show that gapless charged fermionic modes generically lead to imaginary $M_{M}$. 


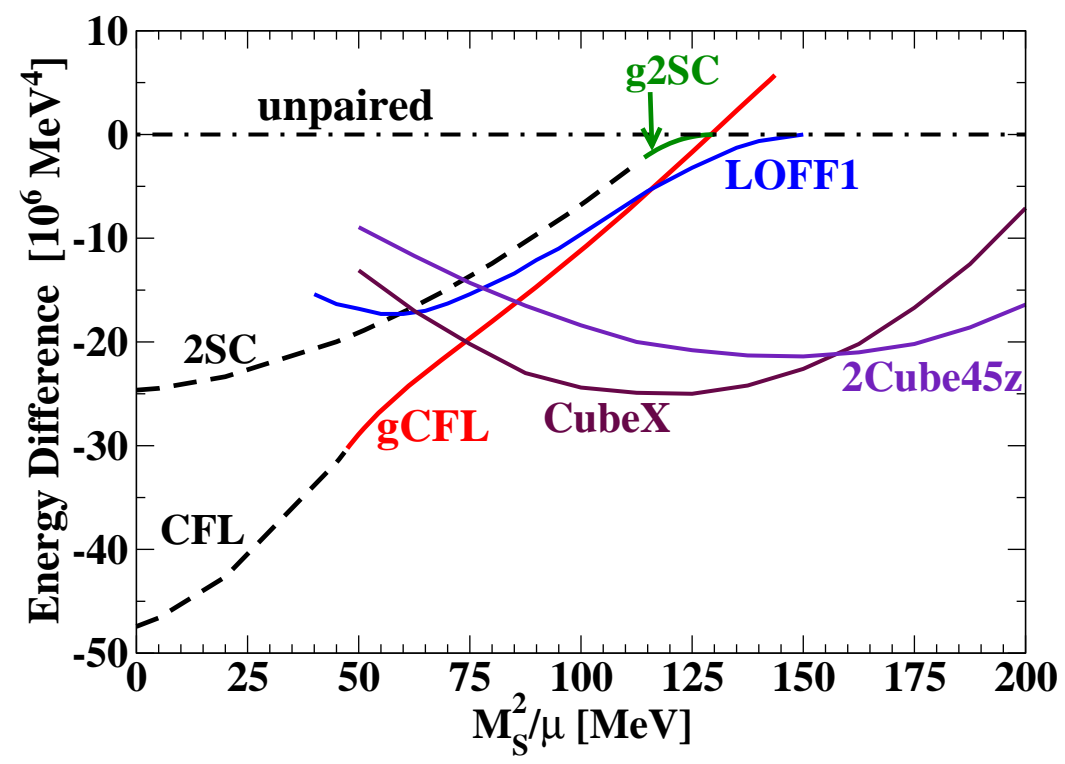

Figure 3: Free energy of various phases of dense QCD. The CFL pairing strength is $\Delta_{C F L}=25 \mathrm{MeV}$. The curves for the CFL, 2SC, gCFL, g2SC, and LOFF1 phases are obtained from an NJL model. Note that the $\mathrm{gCFL}$ phase takes over from CFL at $M_{s}^{2} / \mu \approx 2 \Delta_{C F L}$, and remains favored beyond the value $M_{s}^{2} / \mu \approx 4 \Delta_{C F L}$ at which the CFL phase would become unfavored. The "LOFF1" curve is the single-plane-wave LOFF ansatz of [38]. The "CubeX" and "2Cube $45 z$ " lines are estimates for more complicated LOFF crystal structures, and follow from the Ginzburg-Landau calculation of Ref. [39].

The instability of the gapless phases indicates that there must be other phases of even lower free energy, that occur in their place in the phase diagram. The nature of those phases remains uncertain at present: some candidates are discussed below.

\subsection{Crystalline pairing}

The pairing patterns discussed so far have been translationally invariant. But in the region of parameter space where cross-species pairing is just barely excluded by stresses that pull apart the Fermi surfaces, one expects a position-dependent pairing known as the "LOFF" phase [40, 41, 42, 43]. This arises because one way to achieve pairing between different flavors while accommodating the tendency for the Fermi momenta to separate is to only pair over part of the Fermi surface, and form pairs with non-zero momentum. The LOFF phase therefore competes with the gCFL phase, and may resolve that phase's stability problems.

Recent calculations for 3-flavor quark matter (within a Ginzburg-Landau approximation) show that even a very simple single-plane-wave LOFF ansatz yields a state that has lower free energy than gCFL in the region where the gCFL $\rightarrow$ unpaired transition occurs $[38,44]$ (see Fig. 3) and the Meissner instability is no longer present [45].

The gap parameter and free energy for three-flavor quark matter have also recently been evaluated within a Ginzburg-Landau approximation for many candidate crystal structures [39]. Fig. 3 shows the free energies of the two most favorable crystal structures, CubeX and 2Cube $45 \mathrm{z}$. The robustness of these phases results in their being favored over wide ranges of density. However, it 
also implies that the Ginzburg-Landau approximation is not quantitatively reliable, so the CubeX and 2 Cube $45 \mathrm{z}$ lines in Fig. 3 should only be taken as an indication that the LOFF state might be preferred to gCFL over a much wider range of the stress parameter $M_{s}^{2} /(2 \mu \Delta)$ than one would infer from the single plane wave calculation.

\subsection{Meson supercurrent ("curCFL")}

Kaon condensation alone does not remove the gapless modes that occur in the CFL phase when $M_{s}$ becomes large enough. The CFL- $K^{0}$ phase also develops gapless modes and a Meissner instability, though at a slightly larger value of $M_{s}[46,47]$. One way that the CFL- $K^{0}$ phase can respond is by developing a current in the pseudo-Goldstone bosons (kaons), i.e. a spatial modulation of the $K^{0}$ condensate $[48,49]$. There is no net transfer of any charge because there is a reverse flow in the gapless fermions. The meson current lowers the free energy, but is essentially just another instability: as yet there is no analysis that finds a new meson-supercurrent ground state, whose free energy could be compared with that of other states such as LOFF phases. Calculation of the meson supercurrent in the CFL phase (with no uniform background $K^{0}$ condensate) shows that it is induced when gapless quark modes appear, and that it resolves the Meissner instability, but in that case it is equivalent to a plane-wave LOFF state [50].

\subsection{Gluon condensation}

Analysis of the magnetic instability in the two-flavor gapless color-superconducting phase (g2SC) using a Ginzburg-Landau approach has found that the instability can be cured by the appearance of a chromoelectric condensate [51, 52]. The 2SC condensate breaks the color group down to the $S U(2)_{r g}$ red-green subgroup, and five of the gluons become massive vector bosons via the Higgs mechanism. The new condensate involves some of these massive vector bosons, and because they transform non-trivially under $S U(2)_{r g}$ it now breaks that gauge symmetry. Because they are electrically charged vector particles, rotational symmetry is also broken, and the phase is an electrical superconductor. There are some connections between the gluon condensate and the LOFF phase: the single-plane-wave LOFF state is gauge-equivalent to a homogeneous vector boson condensate. However in general the gluon condensate has non-zero field strength, and is not simply a gauge transformation of an inhomogeneous diquark condensate [35]. In the two-flavor case, gluon condensation appears to be favored over single-plane-wave LOFF [53], but it has not been compared with a LOFF crystal, and as yet the gluon condensate has not been studied in the three-flavor case.

\subsection{Secondary pairing}

Since the Meissner instability is generically associated with the presence of gapless fermionic modes, and the BCS mechanism implies that any gapless fermionic mode is unstable to Cooper pairing in the most attractive channel, one might expect that the instability will simply be resolved by "secondary pairing" of the gapless quasiparticles which would then acquire their own gap $\Delta_{s}$ $[54,55]$. Furthermore, the quadratically gapless mode in gCFL has a greatly increased density of states at low energy (diverging as $E^{-1 / 2}$ ), so its secondary pairing is much stronger than would be predicted by BCS theory: $\Delta_{s} \propto G_{s}^{2}$ for coupling strength $G_{s}$, as compared with the standard 
BCS result $\Delta \propto \exp (-$ const $/ G)$ [54]. This result is confirmed by an NJL study in a two-species model [56], but the the secondary gap was found to be still much smaller than the primary gap, so it does not generically resolve the magnetic instability (in the temperature range $\Delta_{s} \ll T \ll \Delta_{p}$, for example).

\subsection{Single-flavor pairing}

At low enough density, $M_{s}$ puts such a significant stress on the pairing pattern that no pairing between different flavors is possible [22]. The resultant phase is often called "unpaired" quark matter, but there remains the possibility of Cooper pairing where each flavor pairs with itself. (This regime will only arise if $\Delta_{0}$ is so small that very large values of $M_{s}^{2} /\left(\mu \Delta_{0}\right)$ can arise without $\mu$ being taken so small that nuclear matter becomes favored.) Single-flavor pairing may also arise in the 2SC phase, where the strange quarks are not involved in two-flavor pairing. Single-flavor pairing phases have much lower critical temperatures than multi-flavor phases like the CFL or 2SC phases, perhaps as large as a few $\mathrm{MeV}$, more typically in the $\mathrm{eV}$ to many $\mathrm{keV}$ range $[10,11,12,13,57]$, so they are expected to play a role late in the life of a neutron star.

- Single flavor pairing in "unpaired" quark matter. In most NJL studies, matter with no crossspecies pairing at all is described as as "unpaired" quark matter. However, it is well known that there are attractive channels for a single flavor pairing, although they are much weaker than the 2SC and CFL channels [10,11, 12, 13, 57]. Calculations using NJL models and single-gluon exchange agree that the favored phase in this case is the color-spin-locked (CSL) phase [10] in which there is pairing of all three colors of each flavor, with each pair of colors correlated with a particular direction for the spin. This phase does not break rotational symmetry.

- Single flavor pairing in 2SC quark matter. If there is a regime in which the 2SC phase survives, this leaves the blue quarks unpaired. In that case one might expect a " $2 \mathrm{SC}+\mathrm{CSL}$ " pattern, which would again be rotationally symmetric, in which the strange quarks of all three colors self-pair in the CSL pattern. However, the 2SC pattern breaks the color symmetry, and in order to maintain color neutrality, a color chemical potential is generated, which also affects the unpaired strange quarks, splitting the Fermi momentum of the blue strange quarks away from that of the red and green strange quarks (see middle panel of Fig.2). This is a small effect, but so is the CSL pairing gap, and NJL model calculations indicate that the color chemical potential typically destroys CSL pairing of the strange quarks [58]. The system falls back on the next best alternative, which is spin-1 pairing of the red and green strange quarks.

\subsection{Mixed Phases}

Another way for a system to deal with a stress on its pairing pattern is phase separation. In the context of quark matter this corresponds to relaxing the requirement of local charge neutrality, and requiring neutrality only over long distances, so we allow a mixture of a positively charged and a negatively charged phase, with a common pressure and a common value of the electron chemical potential $\mu_{e}$ that is not equal to the neutrality value for either phase. Such a mixture of nuclear and CFL quark matter was studied in Ref. [59]. In quark matter it has been found that as long as we require local color neutrality such mixed phases are not the favored response to the stress imposed by the strange quark mass [27,60]. Phases involving color charge separation have been studied [61] but it seems likely that the energy cost of the color-electric fields will disfavor them. 


\section{Quark matter on the lattice}

For neutron star phenomenology, including color superconductivity in quark matter, the relevant part of the phase diagram is the high-density low-temperature region. Although there has been great progress in mitigating the effects of the sign problem in the complementary region of low chemical potential and high temperature [62, 63, 64], the sign problem remains a severe obstacle to lattice calculations at $\mu \gg T$. It should be noted, however, that the sign problem is more a technical problem than a fundamental one. There is no "no-go" theorem stating that we can never expect to perform lattice QCD calculations at $\mu \gg T$. In certain theories the sign problem has been completely solved, for example the 3-state Potts model [65] and the $O(3)$ model [66], and work is in progress to extend these methods to QCD [67]. It is therefore useful to think about how we would study color superconductivity using lattice QCD if we could perform lattice QCD calculations in the high-density low-temperature region of the phase diagram.

In lattice calculations we have the freedom to vary parameters that are fixed in the real world, such as the number of quark flavors and their masses. Also, issues of electrical charge neutrality and equilibration under the weak interactions do not arise, so lattice QCD would be able to turn off the stresses that were discussed in section 3.1. One significant constraint, however, is the size of the lattice. The size in the Euclidean time direction corresponds to the temperature, and current calculations are limited to sizes less than $5 \mathrm{fm}$, corresponding to $T \geqslant 40 \mathrm{MeV}$. A $20 \mathrm{fm}$ lattice, which is very large by current standards, corresponds to $T=10 \mathrm{MeV}$. Of course, a breakthrough that allows us to work at $\mu \gg T$ may also allow us to work in very large volumes, but the more conservative assumption is that it will remain difficult to study phases with critical temperatures lower than about $10 \mathrm{MeV}$. Superfluidity in nuclear matter, with a critical temperature around 1 $\mathrm{MeV}$, will be therefore be completely inaccessible, and we will have to search for phases with higher critical temperatures. Fortunately, many color superconducting phases are expected to have appropriately high critical temperatures.

\subsection{Quark Matter with $N_{f}$ massless flavors}

In table 1 we give the expected global symmetries of various phases of QCD with $N_{f}=2,3,4$. We do not include $N_{f}=1$ because single-flavor color superconducting phases are predicted to have critical temperatures of order $1 \mathrm{MeV}$ or less $[10,11,12,13]$, so they are not likely to be seen on lattices of a reasonable size. It is noticeable from table 1 that color superconducting phases are not easy to identify. In two-flavor quark matter, the $2 \mathrm{SC}$ color superconductor leaves all the global symmetries unbroken, so there is no order parameter that distinguishes it from unpaired quark matter or quark gluon plasma [16]. In three-flavor quark matter, the CFL color superconductor breaks the global symmetries in exactly the same way as hadronic matter, including complete breaking of the chiral symmetry and superfluidity (since all quarks are massless the baryons are all degenerate, and so the baryon octet can self-pair in a pattern that preserves the flavor symmetry $[68,5])$. In the four-flavor theory, however, Schäfer [6] finds an interesting "partially chirally broken" (P $\chi \mathrm{SB})$ phase, which has different symmetries from any of the other expected phases. Since the staggered fermion formalism naturally yields four continuum flavors, this might be a good place to begin the search for color superconductivity on the lattice. 


\begin{tabular}{|c|c|c|c|}
\hline$N_{f}$ & phase & global symmetry group & description \\
\hline \multirow[t]{4}{*}{2} & unbroken (QGP): & $S U(2)_{L} \otimes S U(2)_{R} \otimes U(1)_{B}$ & \\
\hline & vacuum: & $\rightarrow S U(2)_{V} \otimes U(1)_{B}$ & chiral symmetry broken $(\chi \mathrm{SB})$ \\
\hline & hadronic: & $\rightarrow S U(2)_{V}$ & $\chi \mathrm{SB}$ and superfluid \\
\hline & 2SC: & $\rightarrow S U(2)_{L} \otimes S U(2)_{R} \otimes U(1)_{\tilde{B}}$ & same as QGP \\
\hline \multirow[t]{4}{*}{3} & unbroken (QGP): & $S U(3)_{L} \otimes S U(3)_{R} \otimes U(1)_{B}$ & \\
\hline & vacuum: & $\rightarrow S U(3)_{V} \otimes U(1)_{B}$ & $\chi \mathrm{SB}$ \\
\hline & hadronic: & $\rightarrow S U(3)_{V}$ & $\chi \mathrm{SB}$ and superfluid \\
\hline & CFL: & $\rightarrow S U(3)_{L+R+c} \otimes Z_{2}$ & same as hadronic \\
\hline \multirow[t]{4}{*}{4} & unbroken (QGP): & $S U(4)_{L} \otimes S U(4)_{R} \otimes U(1)_{B}$ & \\
\hline & vacuum: & $\rightarrow S U(4)_{V} \otimes U(1)_{B}$ & $\chi \mathrm{SB}$ \\
\hline & hadronic: & $\rightarrow S U(4)_{V}$ & $\chi \mathrm{SB}$ and superfluid \\
\hline & P $\chi$ SB: & $\rightarrow S U(2)_{V} \otimes S U(2)_{V} \otimes S U(2)_{A}$ & unique [6] \\
\hline
\end{tabular}

Table 1: Symmetry breaking patterns for various phases of QCD with 2,3, and 4 quark flavors. For each $N_{f}$ the last entry is the expected form of color superconductivity at the highest densities.

\subsection{Probing phases and symmetry breaking on the lattice}

There are various tools for to distinguishing different phases of high-density QCD on the lattice.

- Measuring local order parameters. Technically, spontaneous symmetry breaking occurs only in infinite volume systems, where the chance of making transitions between the different possible vacua is zero. More practically, we expect to see spontaneous symmetry breaking when the limit of large volume is taken first, before the limit of taking external currents to zero in the functional integral:

$$
\langle\phi\rangle=\lim _{J \rightarrow 0} \lim _{V \rightarrow \infty} \frac{\delta}{\delta J} \int D \phi \exp (-S[\phi]+J \phi) .
$$

This delicate procedure has been implemented in simpler theories such as the Gross-Neveu model [69]. The order parameter for superfluidity (breaking of $U(1)_{B}$ ) will be a color and flavor singlet dibaryon. The order parameter for chiral symmetry breaking could be the conventional color and flavor singlet chiral condensate, but it is expected that this is suppressed relative to a four-fermion operator $\bar{\psi} \bar{\psi} \psi \psi$ with the same quantum numbers [6].

- Measuring gaps in the fermion spectrum. One of the characteristic consequences of Cooper pairing is the generation of gaps in the fermion spectrum. In color superconducting phases, therefore, we expect to find that the fermionic excitations are classified by representations of the unbroken symmetry group, and that some of them are gapped. In QCD the fundamental fermions are quarks, but gauge invariance dictates that quark "quasiparticles" are still created by baryon creation operators, so the procedure for finding the gaps is the same as that for measuring baryon masses in zero-density QCD. Again, this pairing signature has been seen in the Gross-Neveu model [70], and in the same paper evidence was also found of particle-hole mixing in the fermion spectrum, which is another characteristic of Cooper pairing.

- Measuring low masses of Goldstone bosons. The breaking of a continuous global symmetry, as 
well as giving a non-zero value to some order parameter, creates massless bosonic modes in the spectrum of the theory, known as Goldstone bosons. For chiral symmetry breaking these are the pions. The procedure for measuring the masses is the same as that for measuring meson masses in zero-density QCD.

\section{Conclusion}

As I have described, the project of delineating a plausible phase diagram for real-world highdensity quark matter is still not complete. I have discussed some ideas for the "non-CFL" region of Fig. 1, but there are others such as deformation of the Fermi surfaces (discussed so far only in non-beta-equilibrated nuclear matter [71]) or a Bose-Einstein condensate (BEC) of spatially-bound diquarks [72]. It is very interesting to note that the situation we find in quark matter, a system with pairing that must respond to a stress that separates the chemical potentials of the pairing species, is a very generic one, arising also in condensed matter systems and cold atom systems $[73,74]$. Recent work on BCS/BEC crossover in asymmetric dilute Fermi gases $[75,76,77,78,79,80]$ shows that between the BCS-paired region and the unpaired region in the phase diagram one should expect a translationally-broken region. In QCD this could correspond to a $p$-wave meson condensate, a gluon condensate, or a LOFF state. What is particularly exciting is that the technology of cold atom traps has advanced to the point where fermion superfluidity can now be seen in conditions where many of the important parameters can be manipulated, and it may soon be possible to investigate the response of the pairing to external stress under controlled experimental conditions.

My discussion of lattice approaches to color superconductivity was hypothetical, awaiting a breakthrough that would allow us to evaluate the functional integral at high density and low temperature. Current efforts in this direction include the development of a D-theory formulation that would allow the application of cluster algorithms [67], and also approaches using strong coupling and Hamiltonian methods. In particular, the effective strong-coupling Hamiltonian in the large $N_{c}$ limit has been written as an antiferromagnet with next-to-nearest neighbor couplings, and indications have been found of chiral condensation, but not as yet of quark Cooper pair condensation $[81,82,83,84]$.

At the moment, then, the study of dense quark matter has yielded a diverse landscape of possible phases and phenomenologies across which theorists roam quite freely. Of course there is much interesting work to be done in exploring this territory. But it is also to be hoped that in the future, with increasingly precise observations of neutron star behavior and perhaps even a leap forward in our ability to perform QCD calculations at the relevant densities, we will start to close in on the real geography of the phase diagram of QCD.

\section{References}

[1] J. Bardeen, L. Cooper, J. Schrieffer, Phys. Rev. 106, 162 (1957); Phys. Rev. 108, 1175 (1957)

[2] K. Rajagopal and F. Wilczek, hep-ph/0011333. M. G. Alford, Ann. Rev. Nucl. Part. Sci. 51 (2001) 131 [hep-ph/0102047]. D. K. Hong, Acta Phys. Polon. B 32, 1253 (2001) [hep-ph/0101025]. D. H. Rischke, Prog. Part. Nucl. Phys. 52, 197 (2004) [nucl-th/0305030]. T. Schäfer, hep-ph/0304281. S. Reddy, Acta Phys. Polon. B 33, 4101 (2002) [arXiv:nucl-th/0211045]. 
[3] S. B. Ruster, V. Werth, M. Buballa, I. A. Shovkovy and D. H. Rischke, Phys. Rev. D 72, 034004 (2005) [arXiv:hep-ph/0503184].

[4] M. Alford, K. Rajagopal and F. Wilczek, Nucl. Phys. B537, 443 (1999) [hep-ph/9804403].

[5] T. Schafer and F. Wilczek, Phys. Rev. D 60, 074014 (1999) [arXiv:hep-ph/9903503].

[6] T. Schafer, Nucl. Phys. B 575, 269 (2000) [arXiv:hep-ph/9909574].

[7] I. A. Shovkovy and L. C. R. Wijewardhana, Phys. Lett. B 470, 189 (1999) [arXiv:hep-ph/9910225].

[8] R. D. Pisarski and D. H. Rischke, "Why color-flavor locking is just like chiral symmetry breaking". To be published in, Proceedings of the Judah Eisenberg Memorial Symposium, "Nuclear Matter, Hot and Cold”, Tel Aviv, April 14 - 16, 1999 [nucl-th/9907094].

[9] M. Iwasaki, T. Iwado, Phys. Lett. B350, 163 (1995); M. Iwasaki, Prog. Theor. Phys. Suppl. 120, 187 (1995)

[10] T. Schafer, Phys. Rev. D 62, 094007 (2000) [arXiv:hep-ph/0006034].

[11] M. Buballa, J. Hosek and M. Oertel, Phys. Rev. Lett. 90, 182002 (2003) [arXiv:hep-ph/0204275].

[12] M. G. Alford, J. A. Bowers, J. M. Cheyne and G. A. Cowan, Phys. Rev. D 67, 054018 (2003) [arXiv:hep-ph/0210106].

[13] A. Schmitt, Q. Wang and D. H. Rischke, Phys. Rev. D 66, 114010 (2002) [arXiv:nucl-th/0209050].

[14] K. Iida and G. Baym, Phys. Rev. D 63, 074018 (2001) [Erratum-ibid. D 66, 059903 (2002)] [arXiv:hep-ph/0011229].

[15] M. Alford and K. Rajagopal, JHEP 0206, 031 (2002) [arXiv:hep-ph/0204001].

[16] M. G. Alford, K. Rajagopal and F. Wilczek, "QCD at finite baryon density: Nucleon droplets and color Phys. Lett. B 422, 247 (1998) [arXiv:hep-ph/9711395].

[17] R. Rapp, T. Schafer, E. V. Shuryak and M. Velkovsky, Phys. Rev. Lett. 81, 53 (1998) [arXiv:hep-ph/9711396].

[18] K. Fukushima, C. Kouvaris and K. Rajagopal, Phys. Rev. D 71, 034002 (2005) [arXiv:hep-ph/0408322].

[19] H. Abuki, M. Kitazawa and T. Kunihiro, Phys. Lett. B 615, 102 (2005) [arXiv:hep-ph/0412382].

[20] D. Blaschke, S. Fredriksson, H. Grigorian, A. M. Oztas and F. Sandin, Phys. Rev. D 72, 065020 (2005) [arXiv:hep-ph/0503194].

[21] A. W. Steiner, S. Reddy and M. Prakash, Phys. Rev. D 66 (2002) 094007 [arXiv:hep-ph/0205201].

[22] K. Rajagopal and A. Schmitt, Phys. Rev. D 73, 045003 (2006) [arXiv:hep-ph/0512043].

[23] P. F. Bedaque and T. Schäfer, Nucl. Phys. A 697 (2002) 802 [hep-ph/0105150].

[24] T. Schafer, Phys. Rev. D 65, 094033 (2002) [arXiv:hep-ph/0201189].

[25] M. Buballa, Phys. Lett. B 609, 57 (2005) [arXiv:hep-ph/0410397].

[26] M. M. Forbes, Phys. Rev. D 72, 094032 (2005) [arXiv:hep-ph/0411001].

[27] M. Alford, C. Kouvaris and K. Rajagopal, Phys. Rev. Lett. 92, 222001 (2004) [arXiv:hep-ph/0311286];

[28] R. Casalbuoni, R. Gatto, M. Mannarelli, G. Nardulli and M. Ruggieri, Phys. Lett. B 605, 362 (2005) [Erratum-ibid. B 615, 297 (2005)] [arXiv:hep-ph/0410401]. 
[29] K. Fukushima, Phys. Rev. D 72, 074002 (2005) [arXiv:hep-ph/0506080].

[30] M. Huang and I. A. Shovkovy, Phys. Rev. D 70, 094030 (2004) [arXiv:hep-ph/0408268].

[31] I. Giannakis and H. C. Ren, Phys. Lett. B 611, 137 (2005) [arXiv:hep-ph/0412015].

[32] S. Reddy and G. Rupak, Phys. Rev. C 71, 025201 (2005) [arXiv:nucl-th/0405054].

[33] M. Huang, Phys. Rev. D 73, 045007 (2006) [arXiv:hep-ph/0504235].

[34] K. Iida and K. Fukushima, arXiv:hep-ph/0603179.

[35] M. Hashimoto, arXiv:hep-ph/0605323.

[36] K. Fukushima, Phys. Rev. D 73, 094016 (2006) [arXiv:hep-ph/0603216].

[37] M. Alford and Q. h. Wang, J. Phys. G 31, 719 (2005) [arXiv:hep-ph/0501078].

[38] R. Casalbuoni, R. Gatto, N. Ippolito, G. Nardulli and M. Ruggieri, Phys. Lett. B 627, 89 (2005)

[Erratum-ibid. B 634, 565 (2006)] [arXiv:hep-ph/0507247].

[39] K. Rajagopal and R. Sharma, arXiv:hep-ph/0605316.

[40] A. I. Larkin and Yu. N. Ovchinnikov, Zh. Eksp. Teor. Fiz. 47, 1136 (1964) [Sov. Phys. JETP 20, 762 (1965)]; P. Fulde and R. A. Ferrell, Phys. Rev. 135, A550 (1964).

[41] M. G. Alford, J. A. Bowers and K. Rajagopal, Phys. Rev. D 63, 074016 (2001) [arXiv:hep-ph/0008208].

[42] J. A. Bowers and K. Rajagopal, Phys. Rev. D 66, 065002 (2002) [arXiv:hep-ph/0204079].

[43] R. Casalbuoni and G. Nardulli, Rev. Mod. Phys. 76, 263 (2004) [arXiv:hep-ph/0305069].

[44] R. Casalbuoni, M. Ciminale, R. Gatto, G. Nardulli and M. Ruggieri, arXiv:hep-ph/0606242.

[45] M. Ciminale, G. Nardulli, M. Ruggieri and R. Gatto, Phys. Lett. B 636, 317 (2006) [arXiv:hep-ph/0602180].

[46] A. Kryjevski and T. Schafer, Phys. Lett. B 606, 52 (2005) [arXiv:hep-ph/0407329].

[47] A. Kryjevski and D. Yamada, Phys. Rev. D 71, 014011 (2005) [arXiv:hep-ph/0407350].

[48] T. Schafer, Phys. Rev. Lett. 96, 012305 (2006) [arXiv:hep-ph/0508190].

[49] A. Kryjevski, arXiv:hep-ph/0508180.

[50] A. Gerhold and T. Schafer, Phys. Rev. D 73, 125022 (2006) [arXiv:hep-ph/0603257].

[51] E. V. Gorbar, M. Hashimoto and V. A. Miransky, Phys. Lett. B 632, 305 (2006) [arXiv:hep-ph/0507303].

[52] E. V. Gorbar, M. Hashimoto, V. A. Miransky and I. A. Shovkovy, Phys. Rev. D 73, 111502 (2006) [arXiv:hep-ph/0602251].

[53] O. Kiriyama, D. H. Rischke and I. A. Shovkovy, arXiv:hep-ph/0606030.

[54] D. K. Hong, arXiv:hep-ph/0506097.

[55] M. Huang and I. Shovkovy, Nucl. Phys. A 729, 835 (2003) [arXiv:hep-ph/0307273].

[56] M. Alford and Q. h. Wang, arXiv:hep-ph/0507269.

[57] A. Schmitt, Phys. Rev. D 71, 054016 (2005) [arXiv:nucl-th/0412033];

[58] M. G. Alford and G. A. Cowan, J. Phys. G 32, 511 (2006) [arXiv:hep-ph/0512104]. 
[59] M. G. Alford, K. Rajagopal, S. Reddy and F. Wilczek, Phys. Rev. D 64, 074017 (2001) [arXiv:hep-ph/0105009].

[60] M. Alford, C. Kouvaris and K. Rajagopal, arXiv:hep-ph/0407257.

[61] F. Neumann, M. Buballa and M. Oertel, Nucl. Phys. A 714, 481 (2003) [arXiv:hep-ph/0210078].

[62] O. Philipsen, PoS LAT2005, 016 (2006) [PoS JHW2005, 012 (2006)] [arXiv:hep-lat/0510077].

[63] Z. Fodor and S. D. Katz, "Critical point of QCD at finite T and mu, lattice results for physical JHEP 0404, 050 (2004) [arXiv:hep-lat/0402006].

[64] C. R. Allton et al., "Thermodynamics of two flavor QCD to sixth order in quark chemical Phys. Rev. D 71, 054508 (2005) [arXiv:hep-lat/0501030].

[65] M. G. Alford, S. Chandrasekharan, J. Cox and U. J. Wiese, Nucl. Phys. B 602, 61 (2001) [arXiv:hep-lat/0101012].

[66] S. Chandrasekharan, B. Scarlet and U. J. Wiese, Comput. Phys. Commun. 147, 388 (2002) [arXiv:hep-lat/0110215].

[67] U. J. Wiese, PoS LAT2005, 281 (2006) [arXiv:hep-lat/0510042].

[68] M. G. Alford, J. Berges and K. Rajagopal, Nucl. Phys. B 558, 219 (1999) [arXiv:hep-ph/9903502].

[69] S. Hands and D. N. Walters, Phys. Lett. B 548, 196 (2002) [arXiv:hep-lat/0209140].

[70] D. N. Walters and S. Hands, Nucl. Phys. Proc. Suppl. 129, 554 (2004) [arXiv:hep-lat/0308030].

[71] A. Sedrakian, arXiv:nucl-th/0312053.

[72] A. Sedrakian and J. W. Clark, Phys. Rev. C 73, 035803 (2006) [arXiv:nucl-th/0511076].

[73] W. Zwierlein, C. Stan, C. Schunck, S. Raupach, A. Kerman, and W. Ketterle, Phys. Rev. Lett. 92, $120403(2004)$

[74] G. Partridge, K. Strecker, R. Kamar, M. Jack, and R. Hulet, Phys. Rev. Lett. 95, 020404 (2005)

[75] D. T. Son and M. A. Stephanov, arXiv:cond-mat/0507586.

[76] E. Gubankova, A. Schmitt and F. Wilczek, Phys. Rev. B74, 064505 (2006) [arXiv:cond-mat/0603603].

[77] G. Rupak, T. Schafer and A. Kryjevski, arXiv:cond-mat/0607834.

[78] Y. Nishida and D. T. Son, arXiv:cond-mat/0607835.

[79] A. Bulgac, M. M. Forbes and A. Schwenk, Phys. Rev. Lett. 97, 020402 (2006) [arXiv:cond-mat/0602274].

[80] M. Mannarelli, G. Nardulli and M. Ruggieri, "Evaluating the phase diagram of superconductors with asymmetric spin arXiv:cond-mat/0604579.

[81] B. Bringoltz and B. Svetitsky, Phys. Rev. D 68, 034501 (2003) [arXiv:hep-lat/0211018].

[82] B. Bringoltz and B. Svetitsky, "Spontaneous symmetry breaking in strong-coupling lattice QCD at high Phys. Rev. D 69, 014502 (2004) [arXiv:hep-lat/0310032].

[83] X. Q. Luo and H. S. Chen, Nucl. Phys. Proc. Suppl. 140, 511 (2005) [arXiv:hep-lat/0410027].

[84] Y. Nishida, "Phase structures of strong coupling lattice QCD with finite baryon and Phys. Rev. D 69, 094501 (2004) [arXiv:hep-ph/0312371]. 